ORIGINAL ARTICLE

\title{
Pushing and pulling in association with low back and shoulder complaints
}

\author{
M J M Hoozemans, A J van der Beek, M H W Frings-Dresen, L H V van der Woude, \\ F J H van Dijk
}

Occup Environ Med 2002;59:696-702

See end of article for authors' affiliations

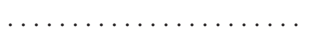

Correspondence to: Dr M H W Frings-Dresen,

Coronel Institute for Occupational and Environmental Health, Academic Medical Centre/University of Amsterdam, PO Box 22700, $1100 \mathrm{DE}$

Amsterdam, Netherlands: m.frings@amc.uva.nl

Accepted 12 March 2002

\begin{abstract}
Aims: To examine the association between exposure to pushing/pulling at work and low back and shoulder complaints.

Methods: A cross sectional questionnaire survey was carried out among 434 workers from several companies with mainly pushing/pulling tasks. From the same companies 188 workers without physically demanding tasks served as reference. The questionnaire was used to assess the exposure to pushing/pulling and other physical risk factors for low back and shoulder complaints. Mean scores at company level were used to classify groups into medium and high exposed to pushing/pulling and a reference group. Psychosocial factors at work were also assessed. Several pain related questionnaires were used to assess the 12 month prevalence of low back and shoulder complaints in three dimensions: trouble (ache, pain, discomfort), pain intensity, and disability. Prevalence rate ratios (PRs) were calculated using Cox's proportional hazards regression analysis.

Results: The 12 month prevalence of low back complaints was almost equal for all three groups. The prevalence of shoulder complaints increased with an increase in exposure level. Adjusted PRs showed that the high exposed group had an increased risk for low back complaints compared to the reference group. For all dimensions of shoulder complaints a clear tendency towards a dose-response relation was observed. The high exposed group had significant PRs for shoulder complaints, ranging from 2.09 to 6.37. Generally, psychosocial factors had a confounding effect for pain intensity and disability, but not for trouble.

Conclusions: For shoulder complaints a dose-response relation was observed for exposure to pushing/pulling. Low back complaints were less consistently associated with pushing/pulling. Stronger associations were observed for more severe cases of low back and shoulder complaints. It is hypothesised that work related psychosocial factors affect these associations.
\end{abstract}

A substantial part of transportation of goods requires manual effort. Lifting loads is increasingly avoided by introducing material handling devices and lifting aids. ${ }^{12}$ Many of these assist devices, however, still require manual pushing and pulling for positioning. Pushing and pulling are also necessary to transport large quantities of packed goods on wheeled carts and are more or less hidden activities of several other tasks, such as patient handling. It is, therefore, not exaggerated when it is stated that overall nearly half of manual materials handling consists of pushing and pulling. ${ }^{3}$

A recent review of epidemiological literature revealed that pushing and pulling is suggested to be a risk factor of musculoskeletal complaints. ${ }^{4}$ Garg and Moore ${ }^{5}$ concluded that pushing and pulling activities accounted for $9-18 \%$ of all back strains and sprains. Of workers' compensation claims, 9\% of all back complaints were associated with pushing or pulling objects, ${ }^{6}$ but the evidence is not highly convincing. ${ }^{7}$ Kuiper and colleagues ${ }^{8}$ reported only one study of sufficient methodological strength that considered pushing and pulling. ${ }^{9}$ They concluded in this systematic review that evidence for development of low back complaints was moderate. In addition to the low back, the shoulders may also be at risk. Pushing and pulling have not been identified as a specific risk factor concerning shoulder complaints. ${ }^{10}$ However, Van der Beek and colleagues ${ }^{11}$ reported an increased risk of shoulder complaints when lorry drivers who regularly pushed or pulled wheeled cages were compared to those who only had a driving task.

Until now, pushing and pulling have not been the primary subject of epidemiological studies and more epidemiological evidence is needed to acknowledge pushing and pulling as risk factors for musculoskeletal complaints. Risk factors for low back and shoulder pain may vary with the definition of low back and shoulder pain. ${ }^{12}{ }^{13} \mathrm{~A}$ clear association between pushing/pulling and musculoskeletal complaints may become apparent in several definitions of pain. Otherwise, it may be hypothesised that pushing and pulling are associated with certain dimensions of complaints only, for example, severity or disability ${ }^{14}$ Therefore, the objective of the present study was to examine the association between pushing/pulling and low back and shoulder complaints among workers in several occupational groups, using several pain related questionnaires.

\section{SUBJECTS AND METHODS \\ Design and study population}

A cross sectional questionnaire survey was carried out among male and female workers. Relatively large companies and institutions, for which it was expected that pushing and pulling was a common activity, were approached to participate in the study. Companies were selected for participation after the approval of the management and after a visual inspection at the work place to ensure that pushing and pulling were prominent activities. Eventually, a company that provides food and drinks in trains, two nursing homes, two flower auctions, a distribution centre of the Dutch postal services, and a large

Abbreviations: $\mathrm{Cl}$, confidence interval; $\mathrm{HWE}$, healthy worker effect; $\mathrm{PR}$, prevalence rate ratio 
Table 1 Numbers of employees of the participating companies that were approached to complete the questionnaire, including employees that regularly pushed or pulled at work (exposed group) and employees without physically demanding tasks (reference group), and the numbers of respondents (response rate percentage) for each category

\begin{tabular}{lcc}
\hline & Population & Response \\
\hline $\begin{array}{l}\text { Rail catering company } \\
\text { Exposed group }\end{array}$ & 97 & $69(71 \%)$ \\
Reference group & 33 & $22(67 \%)$ \\
$\begin{array}{l}\text { Flower auction 1 } \\
\text { Exposed group }\end{array}$ & 77 & $48(62 \%)$ \\
Reference group & 56 & $48(86 \%)$ \\
Flower auction 2 & & \\
Exposed group & 74 & $60(81 \%)$ \\
Reference group & 41 & $35(85 \%)$ \\
Nursing home & & \\
$\quad$ Exposed group & 136 & $121(89 \%)$ \\
Reference group & 109 & $85(78 \%)$ \\
Postal distribution centre & & $68(61 \%)$ \\
Exposed group & 112 & $73(78 \%)$ \\
Refuse collection companies & & $629(76 \%)$ \\
$\quad$ Exposed group & 94 & \\
Total & 829 & \\
\hline
\end{tabular}

group of refuse collectors from several small companies were included in the study. Workers were invited to participate only when they were contracted for an indefinite period of time. Furthermore, within each company workers were included if they regularly performed pushing and pulling tasks or if they performed tasks that were not physically demanding, such as administrative or management tasks. The latter group of workers was included to serve as reference.

All participants were requested to complete a questionnaire. The questionnaire was presented to 590 employees that regularly pushed or pulled at work (exposed group) and to 239 employees without physically demanding tasks (reference group). The employees without physically demanding tasks and 496 employees that regularly pushed or pulled at work received the questionnaire at their home address and were reminded twice, if necessary, within four weeks. Additionally, a group of 94 refuse collectors was directly approached at work to ensure a high response rate. In group sessions they completed the questionnaire in work time. Also, if needed, they were assisted with completing the questionnaire. This more intense treatment was because of an expected relatively low mastery of language compared to the other participants. Table 1 shows the response rate of workers completing the questionnaire in the participating companies. Eventually, 629 questionnaires were completed $(76 \%)$. Of these questionnaires, 622 were found to be filled in correctly and were used for the analyses.

\section{Assessment of independent variables}

Exposure to pushing and pulling was assessed using the Loquest questionnaire ${ }^{15}{ }^{16}$ by asking whether or not pushing or pulling a total weight over $50 \mathrm{~kg}$ was a frequent activity. The answers were assessed on a four point scale (seldom or never, sometimes, quite often, very often). For each of the pushing/pulling groups and non-physically active groups within each company, the percentage of workers scoring within a category of the four point scale was calculated. The percentage was multiplied by one for the seldom or never category to four for the very often category. Hence, a score of 100 was awarded to an occupational group with a 100\% score within seldom or never and a score of 400 to a group with $100 \%$ score within very often. Next, the occupational groups were categorised into three exposure groups: a reference group (100-199 points), a medium exposed group (200-299 points), and a high exposed group (300-400 points). The reference group comprised 188 participants, the medium exposed group 252 participants, and the high exposed group 182 participants. Table 2 shows the demographic characteristics of these exposure groups.

Recent reviews on risk factors for low back and shoulder complaints were screened for potential confounders or effect modifiers in the association between pushing/pulling and low back or shoulder complaints. ${ }^{8}{ }^{10}{ }^{17-23}$ Therefore, again using the Loquest questionnaire, ${ }^{15}{ }^{16}$ the work related physical exposures to working behind a display (long periods of VDU work), working with hands above shoulder height, lifting or carrying loads over $5 \mathrm{~kg}$, driving in vehicles, and bending and/or twisting with the upper body many times per hour were assessed and considered to be potential confounders. For each of the work related physical exposure variables, three exposure groups were constructed following the same procedure as described for pushing and pulling.

While the exposure to these work related physical factors was analysed at group level, the exposure to other potential confounding variables was analysed at individual level. The questionnaire was used to assess gender, age, level of education, years of employment, regular participation in physically demanding sports, and the occurrence of at least one stressful life event during the past 12 months. The psychosocial factors decision authority, skill discretion, psychosocial work demands, coworker support, and supervisor support were assessed using the Questionnaire on Perception and Judgement of Work. ${ }^{24}$ The individual's exposure to the psychosocial factors was dichotomised to high and low exposed using the population's median level as cut off point.

\section{Assessment of dependent variables}

Both shoulder and low back complaints were assessed using two questionnaires. Firstly, a Dutch translation of the

Table 2 Number of respondents of the questionnaire survey, mean age, years of employment in current job, and number of working hours per week for three exposure groups with respect to pushing and pulling over $50 \mathrm{~kg}$

\begin{tabular}{llcc}
\hline & Reference group & $\begin{array}{l}\text { Medium exposed } \\
\text { group }\end{array}$ & $\begin{array}{l}\text { High exposed } \\
\text { group }\end{array}$ \\
\hline Number of participants & 188 & 252 & 182 \\
Men & 94 & 129 & 166 \\
Women & 94 & 123 & 16 \\
Age in years (SD) & $41.1(9.1)$ & $38.0(9.5)$ & $41.2(8.4)$ \\
Years of employment in current job (SD) & $10.7(7.3)$ & $8.6(6.6)$ & $13.9(7.9)$ \\
Working hours per week (SD) & $35(10)$ & $34(11)$ & 38 (4) \\
\hline
\end{tabular}


Table 3 Relative (and absolute) 12 month prevalence of low back and shoulder complaints according to three different dimensions of complaints

\begin{tabular}{|c|c|c|c|}
\hline & $\begin{array}{l}\text { Reference group } \\
(\mathrm{n}=188)\end{array}$ & $\begin{array}{l}\text { Medium exposed } \\
\text { group }(n=252)\end{array}$ & $\begin{array}{l}\text { High exposed } \\
\text { group }(n=182)\end{array}$ \\
\hline \multicolumn{4}{|l|}{ Low back } \\
\hline Trouble (ache, pain, discomfort) & $47 \%(89)$ & $49 \%(123)$ & $48 \%(89)$ \\
\hline High pain intensity & $15 \%(29)$ & $15 \%(38)$ & $21 \%(38)$ \\
\hline High disability & $6 \%(11)$ & $6 \%(14)$ & $12 \%(23)$ \\
\hline \multicolumn{4}{|l|}{ Shoulders } \\
\hline Trouble (ache, pain, discomfort) & $25 \%(48)$ & $27 \%(68)$ & $41 \%(75)$ \\
\hline High pain intensity & $5 \%(9)$ & $9 \%(22)$ & $20 \%(37)$ \\
\hline High disability & $3 \%(5)$ & $6 \%(15)$ & $10 \%(18)$ \\
\hline
\end{tabular}

Standardised Nordic Questionnaire was used for trouble (ache, pain, discomfort) during the past 12 months. ${ }^{25}$ Secondly, pain intensity as well as disability were assessed using the questionnaire described by Von Korff and colleagues. ${ }^{26}$ High pain intensity was defined as an average score of at least five points on the three 10-point scales concerning characteristic pain intensity. An average score of at least five points on the three 10-point disability scales was defined as high disability. An existing Dutch translation of the Von Korff questionnaire was used, which was adjusted such that the 12 month prevalence of high pain intensity and high disability could be assessed.

\section{Analyses and statistics}

Prevalence rate ratios (PRs) and corresponding 95\% confidence intervals (CIs) were calculated using Cox's proportional hazards regression analysis with a constant risk period to study the association between pushing/pulling and low back and shoulder complaints. It has been stated that for estimating risk ratios the estimation of odds ratios by logistic regression would not be a suitable method when prevalences are high, which is the case for musculoskeletal complaints. ${ }^{27}$ In accordance with recent suggestions, Cox's regression was used to estimate PRs, which will give relatively unbiased estimates of the risk ratios while confidence intervals are expected to become too wide. ${ }^{27}{ }^{28}$ For the low back as well as the shoulders, crude PRs between the groups that were medium and high exposed to pushing and pulling and the reference group were calculated for three dimensions of complaints-that is, the 12 month prevalence of trouble, high pain intensity, and high disability.

To prevent collinearity, correlation coefficients between the independent variables were calculated. Working behind display, lifting or carrying loads over $5 \mathrm{~kg}$, and bending and/or

Table 4 Association between low back complaints and exposure to pushing and pulling over $50 \mathrm{~kg}$

\begin{tabular}{|c|c|c|c|}
\hline & $\begin{array}{l}\text { Trouble (ache, pain, } \\
\text { discomfort) }\end{array}$ & High pain intensity & High disability \\
\hline \multicolumn{4}{|c|}{ Crude prevalence rate ratios } \\
\hline Reference group & 1.00 & 1.00 & 1.00 \\
\hline Medium exposed group & 1.04 (0.80 to 1.37$)$ & $0.98(0.61$ to 1.60$)$ & $0.96(0.43$ to 2.11$)$ \\
\hline High exposed group & $1.02(0.76$ to 1.37$)$ & $1.35(0.83$ to 2.18$)$ & 2.15 (1.05 to 4.41$)$ \\
\hline \multicolumn{4}{|c|}{ Adjusted prevalence rate ratios } \\
\hline Reference group & 1.00 & 1.00 & 1.00 \\
\hline Medium exposed group & $1.05(0.78$ to 1.41$)$ & 0.99 (0.59 to 1.66$)$ & $0.83(0.33$ to 2.07$)$ \\
\hline High exposed group & $1.42(0.96$ to 2.10$)$ & 2.15 (1.08 to 4.27$)$ & $2.25(0.71$ to 7.10$)$ \\
\hline \multicolumn{4}{|l|}{ Confounding factors } \\
\hline \multicolumn{4}{|l|}{ Gender } \\
\hline Men & 1.00 & 1.00 & 1.00 \\
\hline Women & 1.45 (1.07 to 1.96$)$ & 1.73 (1.03 to 2.90$)$ & 1.31 (0.58 to 2.99$)$ \\
\hline Age & $1.00(0.99$ to 1.01$)$ & $1.01(0.99$ to 1.03$)$ & 1.01 (0.97 to 1.04 ) \\
\hline \multicolumn{4}{|l|}{ Driving } \\
\hline Reference group & 1.00 & 1.00 & 1.00 \\
\hline Medium exposed & 0.81 (0.56 to 1.17$)$ & $0.68(0.35$ to 1.34$)$ & $0.64(0.21$ to 1.98$)$ \\
\hline High exposed & $0.94(0.61$ to 1.45$)$ & $1.07(0.51$ to 2.27$)$ & $0.90(0.26$ to 3.14$)$ \\
\hline \multicolumn{4}{|l|}{ Decision authority } \\
\hline High & - & - & 1.00 \\
\hline Low & - & - & 1.40 (0.69 to 2.85$)$ \\
\hline \multicolumn{4}{|l|}{ Skill discretion } \\
\hline High & - & - & 1.00 \\
\hline Low & - & - & 1.01 (0.52 to 1.96$)$ \\
\hline \multicolumn{4}{|c|}{ Psychosocial work demands } \\
\hline Low & - & - & 1.00 \\
\hline High & - & - & 2.54 (1.23 to 5.26$)$ \\
\hline \multicolumn{4}{|l|}{ Supervisor support } \\
\hline High & - & - & 1.00 \\
\hline Low & - & - & 1.70 (0.86 to 3.33$)$ \\
\hline
\end{tabular}

Crude and adjusted prevalence rate ratios for pushing and pulling (and $95 \% \mathrm{Cl}$ ) are presented. Prevalence rate ratios were estimated in three separate regression models for each of the three dimensions of complaints. Adjusted prevalence rate ratios were estimated using multivariate models (all included confounding factors and corresponding adjusted prevalence rate ratios are presented in the corresponding column). Gender and age were a priori forced into the models. 
Table 5 Association between shoulder complaints and exposure to pushing and pulling over $50 \mathrm{~kg}$

\begin{tabular}{|c|c|c|c|}
\hline & $\begin{array}{l}\text { Trouble (ache, pain, } \\
\text { discomfort) }\end{array}$ & High pain intensity & High disability \\
\hline \multicolumn{4}{|c|}{ Crude prevalence rate ratios } \\
\hline Reference group & 1.00 & 1.00 & 1.00 \\
\hline Medium exposed group & 1.08 (0.74 to 1.56$)$ & 1.84 (0.85 to 3.99$)$ & 2.25 (0.82 to 6.20$)$ \\
\hline High exposed group & $1.60(1.12$ to 2.31$)$ & $4.22(2.04$ to 8.75$)$ & 3.70 (1.37 to 9.96$)$ \\
\hline \multicolumn{4}{|c|}{ Adjusted prevalence rate ratios } \\
\hline Reference group & 1.00 & 1.00 & 1.00 \\
\hline Medium exposed group & 1.13 (0.78 to 1.64$)$ & 1.97 (0.78 to 5.00$)$ & 3.46 (0.99 to 12.17$)$ \\
\hline High exposed group & 2.09 (1.35 to 3.24$)$ & $6.37(2.36$ to 17.13$)$ & $6.23(1.55$ to 25.13$)$ \\
\hline \multicolumn{4}{|l|}{ Confounding factors } \\
\hline \multicolumn{4}{|l|}{ Gender } \\
\hline Men & 1.00 & 1.00 & 1.00 \\
\hline Women & 2.42 (1.73 to 3.39 ) & $4.16(2.17$ to 7.99$)$ & 3.17 (1.27 to 7.94$)$ \\
\hline Age & 1.01 (0.99 to 1.02$)$ & $0.99(0.96$ to 1.02$)$ & 1.00 (0.97 to 1.04$)$ \\
\hline \multicolumn{4}{|l|}{ Education } \\
\hline High & - & 1.00 & - \\
\hline Low & - & 1.94 (1.09 to 3.46 ) & - \\
\hline \multicolumn{4}{|c|}{ Working above shoulder height } \\
\hline Reference group & 1.00 & 1.00 & 1.00 \\
\hline Medium exposed & 1.82 (1.13 to 2.94$)$ & 2.35 (1.13 to 4.86$)$ & 2.93 (1.03 to 8.35 ) \\
\hline High exposed & - & - & - \\
\hline \multicolumn{4}{|l|}{ Skill discretion } \\
\hline High & - & 1.00 & - \\
\hline Low & - & 1.74 (1.00 to 3.03 ) & - \\
\hline \multicolumn{4}{|c|}{ Psychosocial work demands } \\
\hline Low & - & - & 1.00 \\
\hline High & - & - & 1.46 (0.68 to 3.12$)$ \\
\hline \multicolumn{4}{|l|}{ Coworker support } \\
\hline High & - & 1.00 & 1.00 \\
\hline Low & - & $0.92(0.54$ to 1.58$)$ & 1.30 (0.60 to 2.83 ) \\
\hline \multicolumn{4}{|l|}{ Supervisor support } \\
\hline High & - & - & 1.00 \\
\hline Low & - & - & 1.05 (0.49 to 2.25 ) \\
\hline
\end{tabular}

twisting with the upper body many times per hour, appeared to be highly correlated with pushing and pulling with correlation coefficients of $-0.72,0.65$, and 0.97 , respectively. These variables were subsequently omitted from the epidemiological model as potential confounding factors. Each of the remaining independent variables was screened separately for confounding. Gender and age were selected a priori as confounding factors. The confounding effect of driving a vehicle was examined with respect to low back complaints and working with hands above shoulder height with respect to shoulder complaints. If the PR for pushing and pulling, adjusted for gender and age, was changed at least $10 \%$, the included variable was identified as a confounder and included in the multivariate model. Driving a vehicle appeared to have a confounding effect on low back trouble, pain intensity, and disability. Furthermore, decision authority, skill discretion, psychosocial work demands, and supervisor support changed the PR with respect to low back disability. For shoulder complaints, working above shoulder height appeared to have a confounding effect on all of the three dimensions. The PR for shoulder pain intensity was changed by including education, skill discretion, and coworker support. Psychosocial work demands, coworker support, and supervisor support had a confounding effect on the association between pushing/pulling and shoulder related disability. All statistical analyses were performed with version 9.0.0 of the SPSS statistical package; a significance level of 5\% was used.

\section{RESULTS}

Table 3 presents the 12 month prevalence of low back and shoulder complaints according to the different dimensions of complaints for the three exposure groups concerning pushing and pulling over $50 \mathrm{~kg}$. The prevalence of low back trouble was about $50 \%$ and almost equal for all exposure groups. However, the prevalence of shoulder trouble did increase with an increase in exposure level from $25 \%$ in the reference group to $41 \%$ in the high exposed group. A comparable tendency was observed for the different dimensions of complaints-that is, small differences in prevalence between exposure groups for low back complaints and an increase in prevalence with an increase in exposure for shoulder complaints. With respect to the Von Korff questionnaire, prevalences of high pain intensity and high disability were relatively low.

Tables 4 and 5 confirm the tendency of absolute and relative prevalence rates in complaints observed in table 3 . The crude PRs for the medium and high exposed groups in comparison with the reference group are given in the upper part of tables 4 and 5. Only for low back disability in the high exposed group was there a significant crude PR of 2.15 (95\% CI: 1.05 to 4.41 ) in comparison with the reference group (table 4). The high exposed group showed significant crude PRs for all dimensions of shoulder complaints, ranging from 1.60 for shoulder trouble to 4.22 for high intensity of shoulder pain (table 5).

In table 4 the adjusted PRs are presented for the low back. Generally, only the high exposed group showed an increased risk for low back complaints. For high pain intensity the adjusted PR was statistically significant. Gender appeared to have a significant effect on low back trouble and high pain intensity-women had a significantly higher risk. High psychosocial work demands was a significant risk factor for high disability. 
Table 5 presents the adjusted PRs for shoulder complaints. A clear tendency towards a dose-response relation was observed in all of the three dimensions of shoulder complaints. The high exposed group had a significant PR for all dimensions of shoulder problems, ranging from 2 for shoulder trouble to 6 for the Von Korff measures. The medium exposed group had a marginally significant PR of 3.46 (95\% CI: 0.99 to 12.17) for shoulder disability. Again, when gender was included into the epidemiological model it appeared to have a significant PR for all dimensions of shoulder complaints, with women having the higher risk. For working above shoulder height the medium exposed group showed significant PRs for all dimensions of shoulder complaints in the range 1.82-2.93. Furthermore, low skill discretion and low education were found to be significant risk factors for high intensity of shoulder pain.

\section{DISCUSSION}

The objective of the present study was to examine the association between pushing/pulling and the prevalence of low back and shoulder complaints. Only the group that was highly exposed to pushing and pulling showed an increased risk of low back complaints. However, these increased risks were significant for one of three different dimensions of complaints (table 4). For shoulder complaints a clear tendency towards a dose-response relation was observed. The high exposed group appeared to have a significant increased risk for all studied dimensions of shoulder complaints.

Within the study population selection may have biased results if, for instance, exposed workers who are concerned about their personal health were more likely to respond to the questionnaire survey than other workers. However, it could not be determined whether the response of the questionnaire was liable to selection. Although the overall response can be considered satisfactory (see table 1), some of the companies show a lower than average response. Some of the employees of one of the flower auctions, the postal distribution centre, and the group of refuse collectors were assisted in completing the questionnaire. As the assistance merely consisted of explaining the questions raised in the questionnaire it is not expected that this would have led to biased results, especially with respect to the 12 month prevalence of health complaints.

A healthy worker effect (HWE) might be operating in some or all of the workplaces studied. Because the study presents cross sectional data it is difficult to estimate the impact of the HWE. As a result it is not clear for this study whether workers with complaints tend to leave the highly physical jobs that contain pushing or pulling and whether more workers with complaints have left the job before the start of the study. Assuming that the physically exposed groups are associated with a higher risk of musculoskeletal complaints, the HWE may have caused an underestimation of risks and prevalences. In that case, the true association between pushing and pulling and shoulder complaints will be more pronounced. However, data showed no significant differences in years of employment between workers with and without high pain or disability. If the date of onset of the complaints is known, an indication of the HWE in cross sectional studies can be estimated. ${ }^{29}$ However, such data was not assessed in the present study.

Exposure to pushing and pulling and other work related physical factors was defined at group level. Each person assigned to one of three exposure groups was assumed to have the same mean exposure level. It has been suggested that self reported physical work load may be used to classify occupational groups at an ordinal scale with crude exposure categories. $^{3031}$ Seixas and colleagues ${ }^{32}$ stated that studies aimed at group level are accompanied with essentially unbiased estimates of risk compared to studies aimed at individual level, but group based risk estimates have relatively large random errors. Furthermore, it has been recognised that estimating PRs by Cox's regression would result in more reliable estimates of risk compared to estimating odds ratios by logistic regression when prevalences are relatively high, but that confidence intervals are estimated conservatively. ${ }^{27} 283$ From simulations, Skov and colleagues ${ }^{28}$ concluded that standard errors estimated using Cox's regression seem to be dependent on the prevalence of the outcome measure. For a prevalence of $30 \%$ their data suggested an overestimation of the standard error of $20 \%$ with respect to the true standard error. For a prevalence of $50 \%$ the standard errors could be overestimated by $40 \%$. Thus, from a statistical point of view, the estimated risk levels of the present study can be considered to be relatively unbiased, but confidence intervals are expected to be too wide. Significant associations can, therefore, be considered credible associations.

In the present study the classification of work related physical factors introduced difficulties when eliminating confounding. Exposure groups that represented working behind a display, lifting, and bending/twisting with the upper body were highly correlated with the exposure groups of pushing/ pulling and, therefore, not independent from pushing/pulling. It appears that, at group level, people who push or pull do not work behind displays and their work also contains some lifting and bending/twisting. If the prevalence of shoulder complaints within the reference group is relatively increased because of working behind displays, the PRs of the groups that are medium and high exposed to pushing and pulling with respect to shoulder complaints may even be underestimated. It is expected that lifting loads only has a minor effect. Companies were selected when pushing and pulling were common activities. Only for nurses is it expected that a considerable part of daily work consists of lifting. However, the nurses were assigned to the medium exposed group with respect to pushing and pulling, which, surprisingly, had no increased risk for low back complaints. Bending and twisting of the upper body frequently accompanies pushing or pulling. Therefore, it can still be argued that associations between pushing/pulling and low back complaints may be largely explained by bending and twisting.

To the authors' knowledge pushing and pulling have not been the primary subject of any epidemiological study. Cross sectional associations were reported, usually as part of studies to identify risk factors of low back complaints. Frymoyer and colleagues $^{34}$ reported a significant association between low back pain and pushing/pulling. Damkot and colleagues ${ }^{35}$ calculated a relative objective measure of exposure by multiplying the weight of the pushed objects by the number of pushing efforts required each day. It appeared that the more severe cases of low back pain were associated with significantly higher pushing exposure. In a multivariate analysis to compare groups with different levels of pain, the maximum weight pulled each day and the average weight pulled each day were found to be significant risk factors. Based on workers' compensation claims, Fuortes and colleagues ${ }^{9}$ studied the prevalence of back injuries among nurses using a case-control design. Univariate analysis revealed that only pulling was significantly associated with back injury (odds ratio $1.08,95 \%$ CI: 1.01 to 1.15$)$. Another study reported that pushing or pulling heavy loads more than once a week was found to be a protective factor of sick leave for low back pain, although the association was not significant (odds ratio $0.57,95 \%$ CI: 0.28 to 1.17). ${ }^{13}$ Van der Beek and colleagues ${ }^{11}$ found a significant odds ratio of 1.7 (90\% CI: 1.0 to 3.0) for regular pain or stiffness in the lumbar region, when lorry drivers who also push and pull wheeled cages are compared to lorry drivers who perform hardly any loading/unloading activities. These results are partly confirmed in the present study. Only for regular and/or prolonged low back complaints and for high intensity of low back pain were associations with the high exposed group significant. Discrepancies in results may be explained by the relative large contrast in exposure to pushing/pulling between 
the high exposed group and the reference group. As companies were selected on pushing and pulling in the present study, it can be expected that other studies included workers that may be exposed to pushing and pulling like the medium exposed group. The results of the present study indicate that the association between pushing/pulling and low back complaints is not as strong as could be expected from the literature. On the other hand, the more severe cases of low back pain were associated with pushing and pulling.

To the authors' knowledge, the association between pushing/pulling and shoulder complaints has only been reported by Van der Beek and colleagues. ${ }^{11}$ They found a significant odds ratio of 2.0 (90\% CI: 1.1 to 3.7) with respect to regular pain or stiffness in the shoulder, which is about the same risk ratio as found in the present study. Results point to a stronger association between pushing/pulling and shoulder complaints compared to low back complaints. Pushing/pulling as such can be identified as a specific risk factor for shoulder complaints. Relative higher PRs are found for the more severe cases of shoulder pain and a tendency of an increase in PR with an increase in exposure could be observed. Both point to a possible causal relation, which should be confirmed by longitudinal analysis.

Driving vehicles as well as working above shoulder height appeared to be confounding variables for low back and shoulder complaints, respectively. Working above shoulder height was found to be a significant risk factor for shoulder complaints. Results are confirmed by most of the consulted reviews, as it is generally acknowledged that there is strong evidence with respect to whole body vibration in relation to low back pain, ${ }^{36}$ and sufficient evidence with respect to posture in relation to shoulder pain. Furthermore, women were found to have a significantly increased PR when compared to men for both low back and shoulder complaints. Effect modification of gender, as pointed out by Niedhammer and colleagues, ${ }^{37}$ appeared not to be present. The difference can, therefore, be considered systematic and has been reported independent of occupation. ${ }^{38}$ In a study of pushing and pulling, differences between men and women in exerted forces remained significant when accounting for anthropometry and maximum capacity. It was hypothesised that differences in working methods would explain the findings. ${ }^{39}$ However, De Zwart and colleagues ${ }^{38}$ could not find a single explanation and concluded that gender differences in risk for musculoskeletal complaints should probably be considered multifactorial.

An interesting finding is that the psychosocial factors had a confounding effect only for pain intensity and disability as defined by the Von Korff questionnaire. These questions represent the 12 month prevalence of the more severe cases. The question is whether this is a statistical effect, resulting in the relative wide confidence intervals, or whether psychosocial factors affect the prevalence of reporting more severe pain or disability. Pope ${ }^{40}$ suggested that psychosocial factors intervene in how pain can cause disability, in which case psychosocial factors are intermediate factors and, therefore, cannot be considered confounding factors. Another explanation is suggested by Dionne and colleagues. ${ }^{41}$ They reported an association between education and back related disability, which they explained through somatisation, occupational factors, and lifestyle variables. It is not inconceivable that people with low levels of social support and high psychosocial work demands are more likely to develop pain related disability. The consulted reviews report contradictory results with respect to the relation between physical complaints and psychosocial factors. A likely explanation of the contradictions between reviews is that risk factors of low back pain vary with the definition, ${ }^{12}{ }^{13}$ which is confirmed by the results of the present study. For the study of musculoskeletal complaints in working populations, the Standardised Nordic Questionnaire is widely used and validated. ${ }^{42}{ }^{43}$ The Von Korff questionnaire is

\section{Main messages}

- Work related pushing/pulling is consistently associated with shoulder complaints.

- For shoulder complaints a dose-response relation with pushing/pulling is observed.

- Work related pushing/pulling is not consistently associated with low back complaints.

- Stronger associations between pushing/pulling and low back and shoulder complaints are observed for the more severe cases of complaints.

Policy implications

- Pushing and pulling activities need considerable attention in the design of the working environment to prevent the development of musculoskeletal complaints.

designed for patient based studies and not often applied in working populations. As a result, the number of cases with high pain and disability was very small. The application of the Von Korff questionnaire might be debatable in aetiological studies among working populations. However, this questionnaire is useful in the identification of severe cases and their response to physical work load, which can be important in the prevention of chronic complaints and the evaluation of the effectiveness of occupational rehabilitation.

In conclusion, shoulder complaints appeared to be significantly associated with pushing and pulling. A tendency towards a dose-response relation was observed. Low back complaints were less consistently associated with pushing and pulling. Stronger associations were observed for more severe cases of low back and shoulder complaints. It is hypothesised that work related psychosocial factors may affect these associations. Results should be confirmed by analyses of more objective exposure data and in a longitudinal design.

\section{ACKNOWLEDGEMENTS}

The authors would like to thank TNO Work and Employment for advice and application of the Loquest questionnaire. Josien Coppoolse, Floor Frings, Jitske Groothausen, Jorrit Jansen, Paul Kuijer, Tineke Piersma, Annieck Ricken, Marianne Six Dijkstra, and Marlies Witjes are gratefully acknowledged for data collection and processing. Part of the data was collected and processed in cooperation with the Department of Public Health, Erasmus University of Rotterdam.

\section{Authors' affiliations}

M J M Hoozemans, A J van der Beek, M H W Frings-Dresen, F J H van Dijk, Coronel Institute for Occupational and Environmental Health, AmCOGG Amsterdam Centre for Research into Health and Health Care, Academic Medical Centre/University of Amsterdam, PO Box 22700, 1100 DE Amsterdam, Netherlands

L H V van der Woude, Institute for Fundamental and Clinical Human Movement Sciences, Faculty of Human Movement Sciences, "Vrije Universiteit", Van der Boechorststraat 9, 1081 BT Amsterdam,

Netherlands

\section{REFERENCES}

1 Woldstad JC, Chaffin DB. Dynamic push and pull forces while using a manual material handling assist device. IIE Transactions 1994:26:77-88.

2 Nussbaum MA, Chaffin DB, Stump BS, et al. Motion times, hand forces, and trunk kinematics when using material handling manipulators in short-distance transfers of moderate mass objects. Appl Ergon 2000;31:227-37

3 Baril-Gingras $\mathbf{G}$, Lortie M. The handling of objects other than boxes: univariate analysis of handling techniques in a large transport company. Ergonomics 1995;38:905-25.

4 Hoozemans MJM, Van der Beek AJ, Frings-Dresen MHW, et al. Pushing and pulling in relation to musculoskeletal disorders: a review of risk factors. Ergonomics 1998:41:757-81.

5 Garg A, Moore JS. Epidemiology of low-back pain in industry. Occup Med 1992; 7:593-608. 
6 Klein BP, Jensen RJ, Sanderson LM. Assessment of workers' compensation claims for back strains/sprains. J Occup Med 1984;26:443-8

7 Kelsey JL, Golden AL, Mundt DJ. Low-back pain/prolapsed lumbar intervertebral disc. Rheum Dis Clin North Am 1990;16:699-716.

8 Kuiper JI, Burdorf A, Verbeek JHAM, et al. Epidemiological evidence on manual materials handling as a risk factor for back disorders: a systematic review. Int J Ind Ergon 1999;24:389-404.

9 Fuortes LJ, Shi Y, Zhang M, et al. Epidemiology of back injury in university hospital nurses from review of workers' compensation records and a case-control survey. J Occup Med 1994;36:1022-6.

10 Van der Windt DAWM, Thomas E, Pope DP, et al. Occupational risk factors for shoulder pain: a systematic review. Occup Environ Med 2000;57:433-42.

11 Van der Beek AJ, Frings-Dresen MHW, Van Dijk FJH, et al. Loading and unloading by lorry drivers and musculoskeletal complaints. Int J Ind Ergon 1993;12:13-23.

12 Deyo RA, Battié MC, Beurskens AJHM, et al. Outcome measures for low back pain research. Spine 2000;23:2003-13.

13 Ozguler A, Leclerc A, Landre $M$, et al. Individual and occupational determinants of low back pain according to various definitions of low back pain. J Epidemiol Community Health 2000;54:215-20.

14 Leboeuf-Yde C, Lauritsen JM, Lauritsen T. Why has the search for causes of low back pain largely been non conclusive? Spine 1997;22:877-81.

15 Hildebrandt VH, Douwes M. Physical load and work: questionnaire on musculoskeletal load and health complaints (In Dutch: Lichamelijke belasting en arbeid: vragenlijst bewegingsapparaat). Voorburg Netherlands: Ministerie van Sociale Zaken en Werkgelegenheid, Directoraat-Generaal van de Arbeid, 1991. http://www.arbeid.tno.nl/ kennisgebieden/bewegen_bewegingsapparaat/files/dmq.doc

16 Hildebrandt VH. Prevention of work related musculoskeletal disorders: setting priorities using the standardized Dutch Musculoskeletal Questionnaire. Thesis, Vrije Universiteit Amsterdam, 2001.

17 Bernard BP. Musculoskeletal disorders and workplace factors: a critical review of epidemiological evidence for work-related musculoskeletal disorders of the neck, upper extremity, and low back. Report no. 97-141. Cincinnati: National Institute for Occupational Safety and Health, US Department of Health and Human Services, 1997.

18 Hoogendoorn WE, Van Poppel MNM, Bongers PM, et al. Physical load during work and leisure time as risk factors for back pain. Scand J Work Environ Health 1999;25:387-403

19 Burdorf A, Sorock GS. Positive and negative evidence of risk factors for back disorders. Scand J Work Environ Health 1997;23:243-56.

20 Dempsey PG, Burdorf A, Webster BS. The influence of personal variables on work-related low-back disorders and implications for future research. J Occup Environ Med 1997;39:748-59.

21 Van der Heijden GJMG. Shoulder disorders: a state-of-the-art review. Baillieres Clin Rheumatol 1999:13:287-309.

22 Sluiter JK, Rest KM, Frings-Dresen MHW. Criteria document for evaluation of the work-relatedness of upper extremity musculoskeleta disorders. Scand J Work Environ Health 2001;27(suppl 2): 1-102.

23 Bongers PM, De Winter CR, Kompier MAJ, et al. Psychosocial factors at work and musculoskeletal disease. Scand J Work Environ Health 1993; 19:297-312.
24 Van Veldhoven M. Psychosocial job demands and workstress. Thesis, University of Groningen, 1996. http://www.skb.nl/SKBsite/vbba/ vbba-vragenlijsten. html

25 Kuorinka IAA, Jonsson B, Kilbom A, et al. Standardised Nordic questionnaires for the analysis of musculoskeletal symptoms. Appl Ergon 1987:18:233-7.

26 Von Korff M, Ormel J, Keefe FJ, et al. Grading the severity of chronic pain. Pain 1992;50:133-49

27 Thompson ML, Myers JE, Kriebel D. Prevalence odds ratio or prevalence ratio in the analysis of cross sectional data: what is to be done? Occup Environ Med 1998;55:272-7.

28 Skov T, Deddens J, Petersen MR, et al. Prevalence proportion ratios: estimation and hypothesis testing. Int J Epidemiol 1998;27:91-5.

29 Punnett L. Adjusting for the healthy worker selection effect in cross-sectional studies. Int J Epidemiol 1996;25:1068-76.

30 Viikari-Juntura E, Rauas S, Martikainen R, et al. Validity of self-reported physical work load in epidemiologic studies on musculoskeletal disorders. Scand J Work Environ Health 1996;22:251-9.

31 Burdorf A. Reducing random measurement error in assessing postural load on the back in epidemiologic surveys. Scand J Work Environ Health 1995;21:15-23.

32 Seixas NS, Sheppard L. Maximizing accuracy and precision using individual and grouped exposure assessments. Scand J Work Environ Health 1996;22:94-101.

33 Nurminen $M$. To use or not to use the odds ratio in epidemiologic analyses? Eur J Epidemiol 1995;11:365-71.

34 Frymoyer JW, Pope MH, Costanza MC, et al. Epidemiologic studies of low-back pain. Spine 1980;5:419-23.

35 Damkot DK, Pope MH, Lord J, et al. The relationship between work history, work environment and low-back pain in men. Spine 1984:9.395-9.

36 Bovenzi M, Hulshof CTJ. An updated review of epidemiologic studies on the relationship between exposure to whole-body vibration and low back pain (1986-1997). Int Arch Occup Environ Health 1999;72:351-65.

37 Niedhammer I, Saurel-Cubizolles M, Piciotti M, et al. How is sex considered in recent epidemiological publications on occupational risks? Occup Environ Med 2000;57:521-7.

38 De Zwart BCH, Frings-Dresen MHW, Kilbom Å. Gender differences in upper extremity musculoskeletal complaints in the working population. Int Arch Occup Environ Health 2001;74:21-30.

39 Van der Beek AJ, Kluver BDR, Frings-Dresen MHW, et al. Gender differences in exerted forces and energetic workload during pushing and pulling of wheeled cages by postal workers. Ergonomics 2000;43:269-81.

40 Pope $\mathrm{MH}$. Does manual materials handling cause low-back pain? Int Ind Ergon 1998;22:489-92.

41 Dionne C, Koepsell TD, Von Korff M, et al. Formal education and back-related disability. In search of an explanation. Spine 1995; 20:2721-30.

42 Kaewboonchoo O, Yamamoto H, Miyai N, et al. The standardized Nordic questionnaire applied to workers exposed to hand-arm vibration. J Occup Health 1998;40:218-22.

43 Palmer K, Smith G, Kellingray S, et al. Repeatability and validity of an upper limb and neck discomfort questionnaire: the utility of the standardized Nordic questionnaire. Occup Med 1999;49:171-5. 